\title{
Research on Measurement Method of High Temperature Slag Flow Rate Based on Image Identification
}

\author{
Chong Wang ${ }^{1}$, Hong Wang ${ }^{1,2}$, Xun Zhu ${ }^{1,2}$, Xianyan He ${ }^{1}$, TYu Tan ${ }^{1}$, Bin Ding ${ }^{1}$, Yudong Ding ${ }^{1,2}$ \\ ${ }^{1}$ Institute of Engineering Thermophysics, Chongqing University, Chongqing 400030, China \\ ${ }^{2}$ Key laboratory of Low-grade Energy Utilization Technologies and Systems, Chongqing University, Chongqing \\ 400030, China
}

\begin{abstract}
A method for the measurements of the flow rate of high temperature molten slag using image identification was proposed. Image of molten slag could be acquired by high-speed camera. The flow rate of molten slag was calculated by the diameter, which was obtained by the edge detection, and the velocity of the feature points obtaining by threshold segmentation. Feature points could be found on the liquid column of molten slag by photo graphing, which showed that the method is feasible. Then glycerite was used to study the influences of different shooting parameters on the measurement accuracy. The effects of exposure time, frame rate and focal length on measurement accuracy were obtained. At the same time, it was found that the selection of location and length of feature region would also have a significant impact on the measurement accuracy.
\end{abstract}

Keywords: Flow; Measurement; Algorithm; High Temperature Molten Slag; Image Identification

\section{Introduction}

China is a big country in the steel industry, and its annual steel output accounts for about $50 \%$ of the world's steel output. Blast furnace slag is an important by-product of 35 in the iron and steel industry. It is often used to produce cement clinker and roadbed materials, and has high economic value. In the process of iron and steel smelting, blast furnace slag exists in the form of liquid slag ${ }^{[2]}$ with a temperature as high as $1450-1550^{\circ} \mathrm{C}$. Each ton of slag contains $1770 \mathrm{MJ}^{[3]}$ of heat and a large amount of waste heat resources can be recovered.

At present, the most commonly used method for treating high-temperature slag is water quenching ${ }^{[4]}$ which $^{\left[{ }^{2}\right.}$ consumes a large amount of water resources and cannot utilize the waste heat resources in blast furnace slag, thus there are many shortcomings. The centrifugal granulation waste heat recovery technology has the advantages of saving energy and high waste heat recovery rate ${ }^{[6]}$ and has become one of the most promising methods for treating high-temperature $\operatorname{slag}^{[7]}$. In centrifugal granulation waste heat recovery. In the process, blast furnace slag is discharged from the blast furnace and flows to a high-speed rotating granulation device. After centrifugal crushing, fine solid high-temperature particles are formed, which enter a moving bed for waste heat recovery. Amorphous particles ${ }^{[8-12]}$ are generated in the rapid cooling process for subsequent resource utilization. In this process, it is necessary to ensure that the slag flow rate matches the rotating speed of the granulation device ${ }^{[13]}$ otherwise, the granulation effect will be deteriorated, affecting the waste heat recovery efficiency and subsequent resource utilization. Therefore, reasonable control of the flow of blast furnace slag to ensure its stable flow is the key to the centrifugal granulation waste heat recovery technology. Accurate measurement of high temperature blast furnace slag flow is to maintain its flow quantity stable premise. At present, there are few researches on the detection of high temperature fluid flow. Sun Qiang ${ }^{[14]}$ et al. used the weighing method to find out the relationship between the flow rate and weight of $600{ }^{\circ} \mathrm{C}$ crude zinc liquid and realized the on-line measurement

Copyright (C) 2020 Chong Wang et al.

doi:10.18282/pef.v9i1.849

This is an open-access article distributed under the terms of the Creative Commons Attribution Non-Commercial License (http://creativecommons. org/licenses/by-nc/4.0/), which permits unrestricted non-commercial use, distribution, and reproduction in any medium, provided the original work is properly cited. 
of crude zinc liquid. However, this method needs to additionally set up a weighing device in the system, which increases the complexity of the system. Moreover, the heat dissipation in the measurement process will reduce the temperature of the measured substance and is not suitable for the flow rate of high-temperature slag. Fife ${ }^{[15]}$ et al. use pulsed ultrasonic echo technology to detect echo phase changes of tiny particles in liquid metal and measure liquid flow rate. however, due to the limitation of sensor materials, this method can only be used for liquid metal at most $750{ }^{\circ} \mathrm{C}$ speed detection cannot be applied to flow detection of high-temperature slag; Liu Junjie ${ }^{[16]}$ and others have designed a measuring device for calculating molten iron outflow flow rate by using infrared radiation time difference. This device can be used for molten iron flow rate detection at temperatures as high as $1534{ }^{\circ} \mathrm{C}$. However, this method can only measure the initial velocity of molten iron outflow from the nozzle, and the subsequent flow rate is still unavailable. It is not suitable for long-term operation of slag off-center granulation systems. Bizjan ${ }^{[17]}$ et al. have set up an experimental system suitable for measuring the flow rate of molten rock wool. By calculating the flow velocity of the wave on the fluctuating liquid column, the flow rate of molten rock wool at $1450{ }^{\circ} \mathrm{C}$ is detected, and the measurement error is within $20 \%$.

It requires the liquid column to remain unstable and fluctuating, however, the slag centrifugal granulation system needs to keep the liquid column stable and cannot adopt this method. As mentioned above, there is no mature and reliable method to measure the flow rate of high-temperature slag centrifugal granulation system, and the research in this field is urgently needed.

The image recognition technology ${ }^{[18-20]}$ is a technique for recognizing objects in images to recognize different patterns of targets and objects. It has the advantages of high accuracy and fast recognition speed, and has been widely used in many fields ${ }^{[21-25]}$. Tong Jianjun ${ }^{[26]}$ et al. use image recognition technology to realize the detection of vehicle speed in the monitoring video, and the positioning time reaches about $13 \mathrm{~ms}$, which is accurate.

The rate reached above $92 \%$. Shi Lilian et al. ${ }^{[27,28]}$ used image recognition technology to judge the two-phase flow pattern through threshold segmentation, binarization and other means, and the recognition accuracy rate reached above $85 \%$; He and others have studied the behavior characteristic ${ }^{[29]}$ of molten slag droplets hitting different surfaces by means of gray scale calibration. The above research shows that image recognition technology is likely to become one of the potential feasible technologies for non-contact measurement of high temperature liquid flow.

Therefore, according to the characteristics of slag, this paper designs a high temperature flow detection method based on image recognition. The flow characteristics of high temperature slag are similar to that of Newtonian fluid ${ }^{[30]}$. Therefore, normal temperature glycerin is used as working medium to verify the method, and the influence of shooting parameters and region of interest (ROI) on measurement accuracy is explored.

\section{Experiment and device}

\subsection{High temperature slag shooting verification}

In the centrifugal granulation process of slag, slag will flow from the tundish to the granulation device, forming a liquid column between the two. The change of slag flow rate in this process has obvious influence on granulation quality. However, there is currently a lack of relevant flow measuring equipment to measure high temperature slag. As shown in Figure 1, because the slag granulation process is complicated and the slag flows through the slag chute and other structures during this process, the temperature distribution of the slag liquid flow is not uniform, which makes the slag liquid column present a number of color blocks different from the color of the main liquid column. these color blocks are defined as characteristic points.

Since the high-temperature slag is between $1450-1550{ }^{\circ} \mathrm{C}$, we used a $90 \%$ concentration glycerin solution (Figure 2$)$ close to the viscosity $(=0.344 \mathrm{~Pa} \mathrm{~s})$ of the slag at $1500^{\circ} \mathrm{C}$ as a substitute working medium in carrying out a large number of basic experiments is pre-mixed with a certain amount of carbon powder as a characteristic point in glycerin solution at room temperature to facilitate identification. Comparing Figure 1 with Figure 2 shows that the flow characteristics of the two are very similar.

\subsection{Measurement of molten slag column diameter}

The temperature of blast furnace slag is as high as $1500^{\circ} \mathrm{C}$. It emits strong light and is easy to generate halo in imaging. Therefore, it is necessary to reduce the exposure and take pictures of it in a small aperture mode with low 
exposure time. As shown in Figure 1 in 1.1, the shooting background is difficult to perceive at this time, appearing black, in sharp contrast to bright red liquid column, with clear edge of liquid column. According to the gray gradient change, the position of the edge of the liquid column is judged. It is considered that the molten slag column is a regular cylinder, and the two liquid columns in the image are taken at the shooting angle directly opposite to the liquid column. The distance between the edges is the diameter of the liquid column. Since the method described in this paper is based on image recognition, it is necessary to establish the conversion relation between the pixel length and the actual length in the image.

\subsection{Measurement of slag liquid column velocity}

The RGB color mode is a color standard in industry. Various colors are obtained through the changes of the three color channels of red (R), green $(\mathrm{G})$ and blue (B) and their superposition. RGB is the color representing the three channels of red, green and blue. This standard includes almost all colors that can be perceived by human vision and is one of the most widely used color systems at present.

The color of a computer image can be represented by an RGB value, a reasonable RGB value range is set to judge the characteristic points, the position of the characteristic points in each frame is determined, and the speed of the characteristic points is calculated frame by frame. Assuming that there is no slip between the characteristic point and the main flow of the liquid column, the velocity of the characteristic point can be considered as the velocity of the molten slag liquid column.

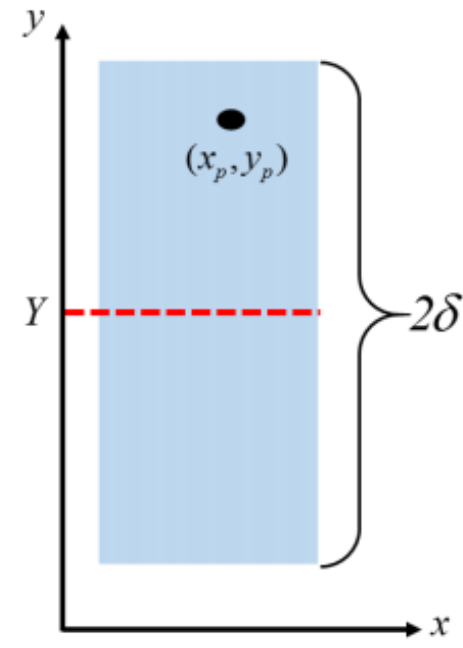

(a) $p$ frame feature point position

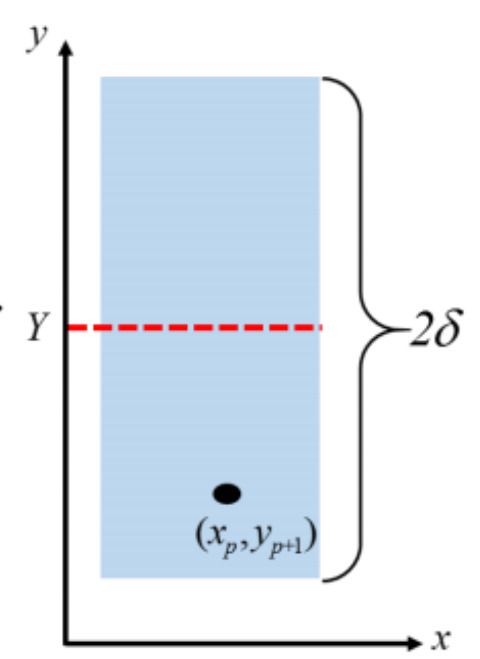

(b) $\mathrm{p}+1$ frame feature point position

Figure3. Algorithm schematic of flow velocity.

\subsection{Experimental system and working conditions}

Figure 4 is a designed and built verification experimental system for slag flow measurement program. The system is mainly composed of an aluminum structure frame $(0.5 \mathrm{~m} \times 0.5 \mathrm{~m} \times 2 \mathrm{~m})$, plexiglass water tank $(0.4 \mathrm{~m} \times 0.3 \mathrm{~m} \times 0.5 \mathrm{~m})$, outflow nozzle, Phantom high-speed camera, tori (tokina) a t-x m100 pro d $100 \mathrm{~mm}$ f2.8macro lens, artificial light source, computer, etc.
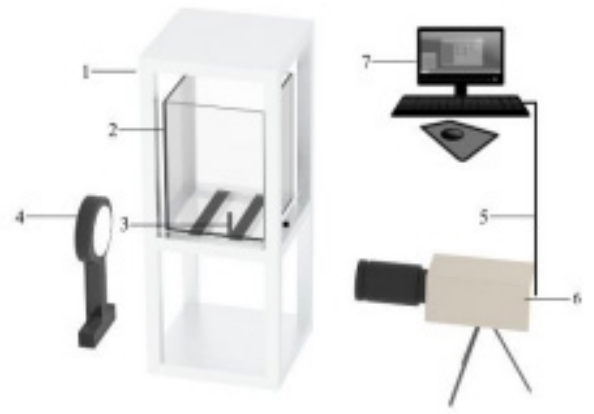

Figure 4. Schematic diagram of experimental system. (1. Acrylic water tank; 2. Aluminum alloy frame; 3. Data. 
connection line; 4. Artificial light source; 5. Data connection line; 6. High speed camera; 7. Computer Diagram)

According to the shape of the liquid column, as shown in Figure 2, the view frame is set to $512 \times 1024$ pixels, with 512 pixels horizontally and 512 pixels vertically pixels.

\section{Experimental results and analysis}

\subsection{Influence of exposure time on measurement accuracy}

In this paper, the glycerin flow rate measured by weighing method is $17.43 \mathrm{~g} \mathrm{~s}-1$, which is taken as the standard flow rate, and the measured value is compared with it. The experiments in this paper were all carried out indoors at 23.4 ${ }^{\circ} \mathrm{C}$ and the measured glycerol density was $=1.21 \mathrm{~g} \mathrm{~cm}-3$ under this condition, and the density was calculated with the subsequent mass flow rate. The exposure time is changed to shoot the glycerin solution liquid column and the obtained image is subsequently processed. Figure 5 shows the relationship between measurement error and exposure time. When the exposure time $\mathrm{t}$ is $200 \mu \mathrm{s}$ false, the error reaches $29.5 \%$, as shown in Figure 5.

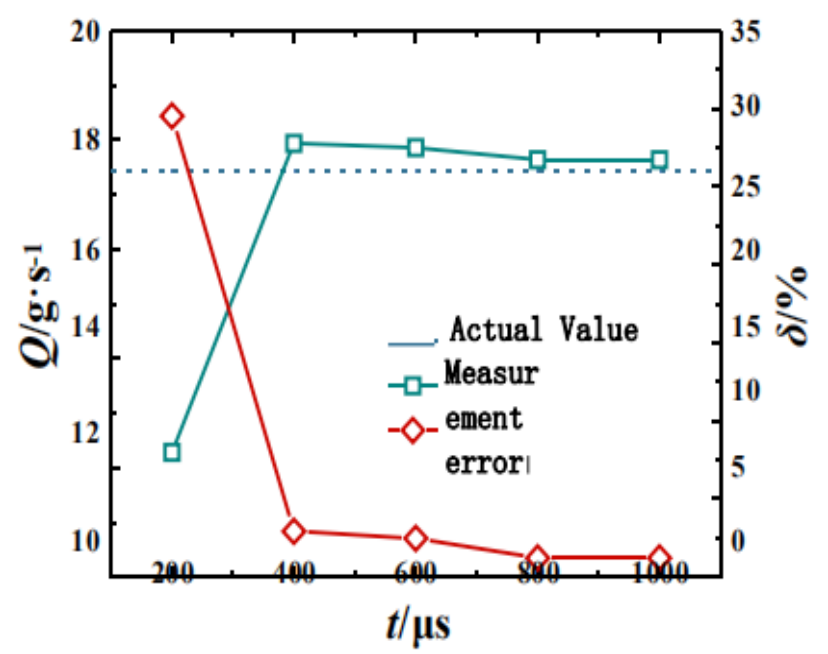

Figure 5. The relation of measurement deviation to exposure time.

\subsection{Influence of shooting frame rate on measurement accuracy}

Changing the shooting frame rate to shoot the glycerin solution liquid column. Figure 6 shows the relationship between measurement error and shooting frame rate. When the shooting frame rate $\mathrm{P}$ is $200 \mathrm{fps}$, the measurement error reaches $15.7 \%$, because the video can be regarded as a collection of several still images, while the shooting frame rate can be regarded as the number of images shot per unit time. When the shooting frame rate P is 200 fps, the number of images taken per unit time is small, and the interval time between each two images is long, which makes the moving distance of the feature point between two consecutive images larger.

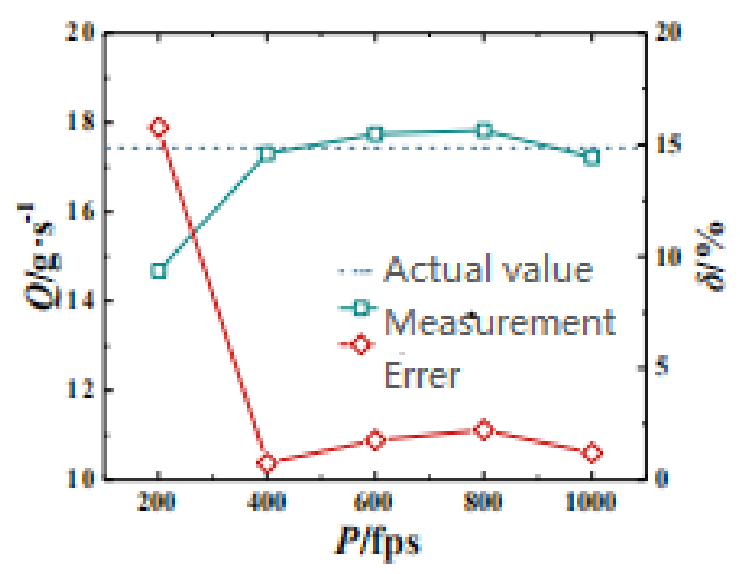

Figure 6. The relation of measurement deviation to frame rate.

\subsection{Influence of material distance on measurement accuracy}


Change the object distance to shoot the glycerin solution liquid column and carry out subsequent processing on the obtained image. Figure 7 shows the relationship between measurement error and object distance u 195. When the object distance $\mathrm{U}$ is $0.4 \mathrm{~m}, 0.5 \mathrm{~m}$ and $0.6 \mathrm{~m}$, the measurement error is only $1.2 \%, 2.1 \%$ and $4.8 \%$, which is because the liquid column occupies a large proportion of the picture, the liquid column contour and characteristic points occupy a large number of pixels, and the identification difficulty is small, so the result is more accurate.

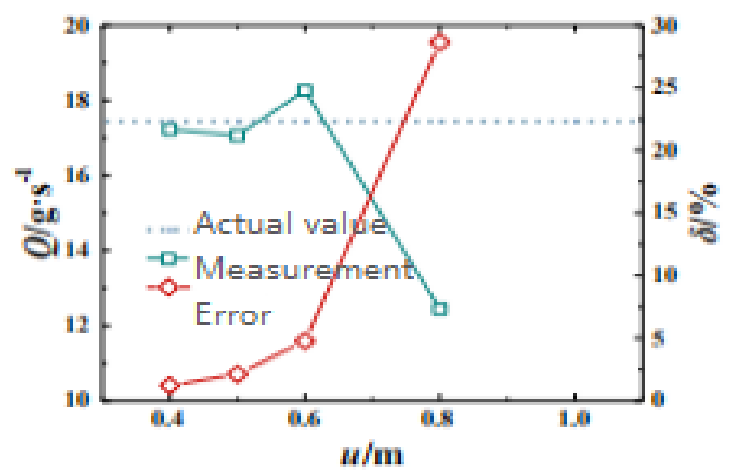

Figure 7. The relation of measurement deviation to focal length.

\section{Conclusion}

(1) Exposure time affects the measurement accuracy by affecting the image contrast. Too low exposure time will cause the characteristic points to be close to the RGB value of the liquid column, thus increasing the measurement error. Too high exposure time will blur the edge and reduce the gray gradient, which is also not conducive to measurement. The shooting frame rate has an optimal value. When shooting at a low frame rate, measurement errors may occur due to horizontal displacement of feature points between two frames, while high frame rate may affect measurement accuracy due to rounding errors. The smaller the object distance is, the more advantageous it is to get the details of the detection area, thus improving the measurement accuracy. For the object distance shot by slag, the high temperature characteristics should be considered and reasonable selection should be made.

(2) When the center position of ROI is too close to the nozzle, the measurement results cannot reflect the actual situation due to the sharp changes in diameter and flow rate. The reasonable ROI position is about 4 times the pipe diameter from the nozzle. The selection of the length of ROI also affects the measurement accuracy. When the selected length of ROI is too small, the feature points pass through ROI too fast and fail to collect enough information for program calculation. The reasonable length of ROI is $1 / 3 \sim 2 / 3$ times the pipe diameter.

(3) The problems of false focus and feature point agglomeration in shooting will bring inevitable errors to the measurement of high-temperature slag, which need to be solved by raising the shooting technology and optimizing the algorithm. In addition, in the actual detection process, problems such as lens deformation, reduction of camera working time and unstable imaging quality are easy to occur, which need to be solved urgently through follow-up research.

\section{References}

1. Wang Wenwen ,Efficiency-based Study on the Impact of China's Iron and Steel Industry Policy,[D] Nanjing: Nanjing University of Technology.2016.

2. Tan Y, Zhu X, Wang H, et al. Centrifugal granulation characteristics of molten blast furnace slag and performance of the granulated particles[J]. Applied Thermal Engineering, 2018, 142: 683-694.

3. Li Q H, Meng A H, Zhang Y G. Recovery status and prospect of low-grade waste energy in China[C]// International Conference on Sustainable Power Generation \& Supply. 2009: 1-6.

4. Comprehensive Utilization of Blast Furnace Slag [J]. Iron and Steel Research, 2010, 38(2): 53-56.

5. Zhang $\mathrm{H}$, Wang $\mathrm{H}$, Zhu X, et al. A review of waste heat recovery technologies towards molten slag in steel industry[J]. Applied Energy, 2013, 112: 956-966.

6. Tan Y, Zhu X, He X Y, et al. Granulation characteristics of molten blast furnace slag by hybrid centrifugal-air blast technique[J]. Powder Technology, 2018, 323: 176-185. 
7. Yang yinkai. Numerical simulation of blast furnace slag dry centrifugal granulation [D]. Wuhan.

8. Ding B, Wang H, Zhu X, et al. Crystallization Behaviors of Blast Furnace (BF) Slag in a Phase-Change Cooling Process[J]. Energy \& Fuels, 2016, 30(4): 3331-3339.

9. Ding B, Zhu X, Wang H, et al. Experimental study on phase change heat transfer characteristics of alloys[J]. International Journal of Heat and Mass Transfer, 2017, 105: 261-269.

10. Qiu Yongjun, Zhu Xun, Wang Hong, et al. Three-dimensional Numerical Simulation of Air Cooling

11. Wang H, Ding B, Zhu X, et al. Influence of Al2O3 content on crystallization behaviors of blast furnace slags in directional solidification process[J]. International Journal of Heat and Mass Transfer, 2017, 113: 286-294.

12. Memon S A, Lo T Y, Barbhuiya S A, et al. Development of form-stable composite phase change material by incorporation of dodecyl alcohol into ground granulated blast furnace slag[J]. Energy and Buildings, 2013, 62:360367.

13. Du Bin, Zhang Yanguo. Experimental Study on Centrifugal Granulation of Liquid Blast Furnace Slag by Rotating Disk [J]. Metallurgical Energy, 2013, 32(4): 29-32.

14. Sun Qiang, Gui Weihua, Li Yonggang, et al. A new method for on-line detection of high temperature fluid flow [J]. Computing Technology and Automation, 2001, 20(4).10-13.

15. Fife S , Andereck C D, Rahal S. Ultrasound thermometry in transparent and opaque fluids[J]. Experiments in Fluids, 2003, 35(2):152-158.

16. Bizjan B, Širok B, Chen J. Optical measurement of high-temperature melt flow rate[J]. Applied Optics, 2018, 57(15): 4202-4210.

17. Liu Junjie. Study on dynamic vertical velocity detection of molten iron with infrared radiation time difference [D]. Shenyang: Northeastern University.2010.

18. Qu Ningning, Cai Xiaoshu, Zhou Zhi, et al. Experimental measurement of coherent structure of turbulent boundary layer by moving single frame image method [J]. Journal of Chemical Engineering, 2017(11):104-110.

19. Yan Z Y, Yan M, Hao S, et al. Cloud and Cloud Shadow Detection Using Multilevel Feature Fused Segmentation Network[J]. IEEE Geoscience and Remote Sensing Letters, 2018, 15(99): 1600-1604.

20. Sun Peng, Chai Tianyou, Zhou Xiaojie, et al. Flame Image Recognition System in Alumina Rotary Kiln [J]. Journal of Chemical Engineering, 2008, 59(7).

21. Romdhani S, Blanz V, Vetter T. Face Identification by Fitting a 3D Morphable Model Using Linear Shape and Texture Error Functions[C]// Computer Vision - ECCV 2002. Springer Berlin Heidelberg, 2002: 3-19.

22. Jassim E W, Newell T A, Chato J C. Probabilistic determination of two-phase flow regimes in horizontal tubes utilizing an automated image recognition technique[J]. Experiments in Fluids, 2007, 42(4): 563-573.

23. Anthonys G, Wickramarachchi N. An image recognition system for crop disease identification of paddy fields in Sri Lanka[C]2009 International Conference on Industrial and Information Systems (ICIIS). 2009: 403-407.

24. Figueiredo M M F, Goncalves J L, A. M. V. Nakashima, et al. The use of an ultrasonic technique and neural networks for identification of the flow pattern and measurement of the gas volume fraction in multiphase flows[J]. Experimental Thermal and Fluid Science, 2016, 70: 29-50.

25. Yin X X, Hadjiloucas S, Zhang Y, et al. Pattern identification of biomedical images with time series: Contrasting THz pulse imaging with DCE-MRIs[J]. Artificial Intelligence in Medicine, 2016, 67: 1-23.

26. Tong Jianjun, Zou Mingfu. Vehicle Speed Measurement Based on Surveillance Video Images [J]. journal of image and graphics, 2005, 10(2): 192-196.

27. Shi Lilian, Zhou zekui, ren shapu. image detection method of gas-liquid two-phase flow parameters in vertical pipeline [J]. fluid machinery, 2004, 32(9): 4-6.

28. Shi Lilian, Cai Jinhui, Zhou Zekui. Identification of gas-liquid two-phase flow patterns based on image processing [J]. Journal of Zhejiang University (Engineering Edition), 2005, 39(8). 1128-1131.

29. He X Y, Zhu X, Wang H, et al. Experimental visualization and theoretical analysis of the dynamic impact behavior of a molten blast furnace slag droplet on different surfaces[J]. Applied Thermal Engineering, 2019, 147: 1-9.

30. Wu Junjun, Wang Hong, Zhu Xun, et al. Filamentous granulation characteristics in rotary centrifugal granulation [J]. Journal of Chemical Engineering, 2015, 66(7): 2474-2480. 